\title{
Probing the oligomeric state and interaction surfaces of Fukutin-I in dilauroylphosphatidylcholine bilayers
}

\author{
P. Marius • Yuk Ming Leung • T. J. Piggot • \\ S. Khalid $\cdot$ P. T. F. Williamson
}

Received: 8 August 2011/Revised: 10 October 2011/Accepted: 24 October 2011/Published online: 11 November 2011

(C) The Author(s) 2011. This article is published with open access at Springerlink.com

\begin{abstract}
Fukutin-I is localised to the endoplasmic reticulum or Golgi apparatus within the cell, where it is believed to function as a glycosyltransferase. Its localisation within the cell is thought to to be mediated by the interaction of its $\mathrm{N}$-terminal transmembrane domain with the lipid bilayers surrounding these compartments, each of which possesses a distinctive lipid composition. However, it remains unclear at the molecular level how the interaction between the transmembrane domains of this protein and the surrounding lipid bilayer drives its retention within these compartments. In this work, we employed chemical cross-linking and fluorescence resonance energy transfer measurements in conjunction with multiscale molecular dynamics simulations to determine the oligomeric state of the protein within dilauroylphosphatidylcholine bilayers to identify interactions between the transmembrane domains and to ascertain any role these interactions may play in protein localisation. Our studies reveal that the N-terminal transmembrane domain of Fukutin-I exists as dimer within dilauroylphosphatidylcholine bilayers and that this interaction is driven by interactions between a characteristic TXXSS motif. Furthermore residues close to the N-terminus that have previously been shown to play a key role in
\end{abstract}

Electronic supplementary material The online version of this article (doi:10.1007/s00249-011-0773-5) contains supplementary material, which is available to authorized users.

P. Marius · P. T. F. Williamson $(\square)$

Centre for Biological Sciences, Highfield Campus,

University of Southampton, Southampton SO17 1BJ, UK

e-mail: P.T.Williamson@ soton.ac.uk

Y. M. Leung $\cdot$ T. J. Piggot $\cdot$ S. Khalid $(\bowtie)$

School of Chemistry, Highfield Campus,

University of Southampton, Southampton SO17 1BJ, UK

e-mail: S.Khalid@soton.ac.uk the clustering of lipids are shown to also play a major role in anchoring the protein in the membrane.

Keywords Fukutin - Glycosyltransferases - Membranes · Solid-state NMR · Molecular dynamics

\section{Introduction}

Dystroglycan is an integral part of the dystroglycan-glycoprotein complex that is responsible for linking the cell's cytoskeleton to the surrounding extracellular matrix. The integrity of this complex has been shown to be dependent on correct glycosylation of dystroglycan (Martin-Rendon and Blake 2003). Hypoglycosylation of dystroglycan has been shown to lead to a family of muscular dystrophies, collectively known as dystroglycanopathies, in which patients exhibit muscle cell instability and defects in neuronal migration (Martin-Rendon and Blake 2003). Dystroglycanopathies have been linked to six genes, including Fukutin, Fukutin Related Protein (FKRP), POMGnT1, POMT1, POMT2 and LARGE (Martin-Rendon and Blake 2003). Sequence analysis of this family of genes suggests that they all encode type II integral membrane proteins which possess putative or demonstrated glycosyltransferase activity in keeping with their role in the O-linked glycosylation of dystroglycan (Torelli et al. 2005; KeramarisVrantsis et al. 2007; Matsumoto et al. 2004; Lommel et al. 2008). A number of studies have demonstrated that the proteins encoded by these genes are localised to the endoplasmic reticulum (ER) or Golgi apparatus (GA) within the cell (Torelli et al. 2005; Keramaris-Vrantsis et al. 2007; Matsumoto et al. 2004; Lommel et al. 2008). Interestingly, a number of mutations identified in these genes lead to mislocalisation of the protein within the cell, 
suggesting that their retention within the correct compartments of the GA/ER is vital for appropriate glycosylation of dystroglycan (Keramaris-Vrantsis et al. 2007).

The localisation of proteins within the ER/GA is a highly dynamic process that relies on tight regulation of antero- and retrograde transport steps. It is widely acknowledged that retrograde transport is largely regulated by receptor-mediated recognition of specific extra-membranous motifs that are found in ER/GA-resident proteins (Gleeson 1998). In contrast, anterograde transport appears to be dependent on the shorter transmembrane domains that are typically found in ER/GA-resident proteins (Gleeson 1998; Pelham and Munro 1993). Indeed, it has been demonstrated that, in the case of Fukutin-I and Fukutin Related Protein, their shorter N-terminal transmembrane domains are sufficient to bring about their retention within the ER/GA (Esapa et al. 2005). Although it is clear that the $\mathrm{N}$-terminal transmembrane domains of ER/GA-resident proteins are responsible for their localisation, we are far from a molecular understanding of this process. It has been suggested that interactions between the protein's shorter $\mathrm{N}$-terminal transmembrane domain and the lipid bilayers surrounding these compartments, with their characteristic physical and chemical properties, may play an important role in retaining proteins within these compartments (Opat et al. 2001; Pelham and Munro 1993; Munro 1998; Rayner and Pelham 1997). At the molecular level, these models suggest that the lipid bilayer properties may alter either the lateral segregation of the protein (the so-called lipid sorting model) or the oligomeric state of the protein within the bilayer. Both models have been proposed to play an important role in regulation of onward trafficking of proteins from these compartments (Opat et al. 2001; Pelham and Munro 1993; Munro 1998; Rayner and Pelham 1997).

To determine the relevance of these models to the retention of the Fukutin family of putative glycosyltransferases within the ER/GA, we are investigating how the lipid composition affects the structure, oligomeric state and lateral segregation of the $\mathrm{N}$-terminal transmembrane domains of this family of proteins. Here we focus on the transmembrane domain of the protein encoded by Fukutin-I (hereinafter referred to as FK1TMD), whose mislocalisation has been linked to the onset of Fukuyama muscular dystrophy. Our earlier studies have revealed that, in response to changes in bilayer thickness, FK1TMD tilts within the bilayer to avoid hydrophobic mismatch, and we have identified a number of lipid-protein interactions responsible for anchoring the protein within the bilayer (Holdbrook et al. 2010; Marius et al. 2010). To ascertain the oligomeric state in bilayers of a similar thickness to those found in the ER/GA, we have undertaken a combined experimental and computational study of FK1TMD reconstituted into dilauroylphosphatidylcholine (DLPC) bilayers. Although representing a simplification of the membranes found in the ER/GA, the thickness of DLPC bilayers (Gallova et al. 2004) is similar to that reported from pure lipid extracts obtained from these organelles (Mitra et al. 2004). The studies presented here have enabled us to ascertain that, in bilayers whose thickness mimics that found in the ER/GA, FK1TMD exists predominantly as a dimeric species. Furthermore, computational studies have enables us to identify the interactions that drive the formation of the dimer and how this affects the protein's interaction with the surrounding bilayer.

\section{Materials and methods}

The N-terminus of Fukutin, FK1TMD (MQRINKNVVL ALLTLTSSAF LLFQLYYYKH YLSARN), was custom synthesised by PeptideSynthetics (Fareham, UK). The peptide was used as supplied at $>70 \%$ purity and gave a single species of appropriate molecular weight when analysed by mass spectrometry. The lipid 1,2-dilauroyl$s n$-glycero-3-phosphocholine (DLPC) was ordered from Avanti Polar Lipids (Alabaster, USA). Fluorescein isothiocyanate (FITC), Rhodamine B isothiocyanate (RBITC), glutaraldehyde and disuccinimidyl suberate (DSS) were ordered from Sigma. Reagents for buffers and gels were also purchased from Sigma.

\section{Cross-linking of reconstituted FK1TMD}

To reconstitute FK1TMD peptide into DLPC vesicles, $20 \mu \mathrm{g}$ peptide (from stock of $1 \mathrm{mg} / \mathrm{ml}$ in methanol) was mixed with DLPC (from a stock $10 \mathrm{mg} / \mathrm{ml}$ solution) at lipid-to-peptide molar ratio of 100:1. The sample was dried overnight under vacuum to remove the solvent. The lipidpeptide film was then resuspended in $100 \mu \mathrm{L} 10 \mathrm{mM}$ sodium phosphate buffer, $\mathrm{pH}$ 7.4. The resulting suspension was then sonicated to clarity for $20 \mathrm{~min}$ (Grants Ultrasonic Waterbath), resulting in the formation of small unilamellar vesicles. The cross-linking experiment was carried out by incubating $10 \mu \mathrm{g}$ reconstituted FK1TMD with $180 \mu \mathrm{g}$ DSS [from stock solution of $90 \mu \mathrm{g} / \mu \mathrm{l}$ in dimethyl sulphoxide (DMSO)] or $0.05 \%$ glutaraldehyde. This was mixed and incubated at room temperature for $1 \mathrm{~h}$. The reaction was stopped by addition of gel loading buffer. Samples were then run on a $16 \%$ tricine gel and stained with SYPRO Orange (Sigma) according to manufacturer's protocol.

Preparation of fluorescently labelled FK1TMD

The free amine groups within FK1TMD $(5 \mathrm{mg})$ were reacted with 3 molar excess of either FITC or RBITC (from DMSO stock solution) in $30 \mathrm{mM}$ solution of pyridine in acetonitrile. 
The labelling reaction was left overnight at room temperature in the dark. Unlabelled peptide was removed by passage of the mixture through a 10-mL column of medium-grade Biogel-P 6 resin (Bio-Rad) equilibrated with buffer A [10 mM 4-(2hydroxyethyl)-1-piperazineethanesulphonic acid (HEPES), $1 \mathrm{mM}$ ethylene glycol tetraacetic acid (EGTA), pH 7.4] containing 5\% acetonitrile. Eluted sample was diluted in an equal volume of water and spun at 13,000 rpm for $5 \mathrm{~min}$. The pellet was lyophilised and then resuspended in methanol. The final labelling ratios were determined by using the following extinction coefficients: FITC, $\varepsilon(494 \mathrm{~nm})=70,000 \mathrm{M}^{-1}$ $\mathrm{cm}^{-1}$; RBITC, $\varepsilon(555 \mathrm{~nm})=103,000 \mathrm{M}^{-1} \mathrm{~cm}^{-1}$; FK1TMD, $\varepsilon=5,960 \mathrm{M}^{-1} \mathrm{~cm}^{-1}$. Molar labelling ratio of dye to peptide was approximately $1: 1$.

\section{Reconstitution of labelled peptide}

Mixtures of FITC, RBITC and unlabelled FK1TMD were reconstituted into DLPC vesicles at lipid-to-protein ratio of 100:1 by mixing $10 \mu \mathrm{mol}$ DLPC with 100 nmoles total peptide. The resulting mixture was dissolved in methanol to ensure even mixing of all components, dried to a thin film and left under high vacuum overnight to remove any residual organic solvent. The film was hydrated and suspended in $500 \mu \mathrm{l}$ buffer $\mathrm{A}$, freeze-thawed three times and then sonicated to clarity for $2 \mathrm{~min}$. The concentration of the donor FITC-labelled FK1TMD was fixed for each sample, with the remaining peptide being made up of unlabelled and acceptor RBITC-labelled FK1TMD at varying ratios. The ratio of FITC-labelled to RBITClabelled and unlabelled peptide was fixed at 1:3.

\section{Förster resonance energy transfer (FRET)}

FRET measurements were carried out at room temperature in triplicate using a Hitachi F-2500 spectrofluorometer interfaced with a computer for data accumulation. Lipid vesicles containing known concentrations of FITC- and RBITC-labelled peptide were used for experimentation. In a typical experiment, $250 \mu \mathrm{l}$ reconstituted peptide was diluted in buffer A to concentration of $22 \mu \mathrm{M}$ in an ultraviolet (UV)-grade cuvette (Fisher). Samples were excited at wavelength of $492 \mathrm{~nm}$, and emission was monitored from 500 to $650 \mathrm{~nm}$ at scan rate of $300 \mathrm{~nm} / \mathrm{min}$. Excitation and emission slit widths were set to $10 \mathrm{~nm}$. The FITC fluorescence intensity was determined at $520 \mathrm{~nm}$ in the presence and absence of RBITC. The reduction in FITC emission intensity was used as a measure of energy transfer. Emission spectra were corrected for scattering from vesicles by the subtraction of an appropriate spectrum of unlabelled vesicles. No corrections were made for RBITC, as it makes no contribution to the emission spectra at $520 \mathrm{~nm}$ when excited at $492 \mathrm{~nm}$.
Molecular dynamics

\section{Generation of FK1TMD model}

A model of the 36-residue transmembrane domain of Fukutin-I (FK1TMD) was created by threading the FK1TMD sequence to an idealised $\alpha$-helix using Modeller 9v7 (Marti-Renom et al. 2000). The resulting helix was evaluated for stereochemical integrity using Procheck (Laskowski et al. 1993). This model was used for all of the atomistic simulations and also for creating the coarsegrained version of the FK1TMD.

The atomistic model of the dimer was generated from the coarse-grained (CG) model using the protocol described by Stansfeld and Sansom (2011). The DLPC lipids were mapped from CG to atomistic (AT) resolution using our in-house reverse-mapping tool, which constructs the positions of the individual lipid atoms based solely on the CG geometry.

\section{Details of atomistic simulations}

Atomistic simulations were performed using GROMACS version 4.5.1 (Berendsen et al. 1995; van der Spoel et al. 2005; Hess et al. 2008). The simulations used the unitedatom GROMOS 53A6 force field (Oostenbrink et al. 2004) with DLPC lipid parameters adapted from the dipalmitoylphosphatidylcholine (DPPC) parameters provided by Kukol (2009). Water molecules were treated explicitly using the simple point charge (SPC) water model (Berendsen et al. 1981). Sodium and chloride ions were added at concentration of $1 \mathrm{M}$. All lipid and protein bonds were constrained using the LINCS algorithm (Hess 2008), and water molecules were constrained using the SETTLE algorithm (Miyamoto and Kollman 1992), allowing for a time step of 2 fs to be used. A Nosé-Hoover thermostat, with time constant for the coupling of $0.5 \mathrm{ps}$, was used to maintain the system temperature (Hoover 1985). A Parinello-Rhaman barostat, with time constant of $5.0 \mathrm{ps,} \mathrm{was}$ used to maintain the system pressure at 1.0 bar (Parrinello and Rahman 1981). Electrostatic interactions used a cut-off of $0.9 \mathrm{~nm}$ with interactions beyond this cut-off treated using the smooth particle mesh Ewald (PME) method (Essmann et al. 1995). The van der Waals interactions used a cut-off of $1.4 \mathrm{~nm}$ with a long-range dispersion correction applied for the energy and pressure. The neighbour list was updated every five steps. The system components of each simulation are summarised in Supplementary Table 1. All simulations were run for $100 \mathrm{~ns}$. The conformational properties of FK1TMD were analyzed using GROMACS tools and the DSSP program (Kabsch and Sander 1983). Visualisation used VMD (Humphrey et al. 1996). 


\section{Details of coarse-grained simulations}

All CG simulations were performed using GROMACS 4.5.1 (15-17) with the MARTINI CG force fields. All simulations involved self-assembly of a lipid bilayer from a random configuration of lipids, ions and water as described by Marrink et al. (2004), Bond and Sansom (2006) and Carpenter et al. 2008). The FK1TMD molecules were then inserted into the assembled membranes so that the two monomers were the maximum distance apart. The CG parameters for DLPC, ions and water molecules were as described by Marrink et al. (2004). Parameters for amino acids were as described by Bond and Sansom (2006) and Bond et al. (2007, 2008). The integrity of the FK1TMD helix was retained by implementing an elastic network model as described by Bond et al. (2007). As with the atomistic simulations, analyses of the CG simulations were performed using GROMACS tools and locally written code and visualisation used VMD (Humphrey et al. 1996).

\section{Results and discussion}

\section{Chemical cross-linking}

To determine the self-association of FK1TMD and ascertain its oligomeric state in DLPC bilayers, crosslinking experiments were conducted using $0.45 \%$ DSS, a membrane-permeable cross-linking reagent that reacts with primary amines. In the absence of DSS, FK1TMD runs as a single species on sodium dodecyl sulphate (SDS) gel (Fig. 1) with apparent molecular weight of approximately $4 \mathrm{kDa}$, in close agreement with the predicted mass of 4,294 Da. Importantly, this demonstrates that, in the presence of the detergent SDS, FK1TMD runs as a monomeric species. Treatment of the FK1TMD reconstituted into DLPC vesicles with DSS resulted in two species on the SDS gel: the first at approximately $4 \mathrm{kDa}$ reflecting the presence on monomeric FK1TMD, and a second at $8 \mathrm{kDa}$ arising from a dimeric population of FK1TMD within the membrane which had presumably been cross-linked by DSS via either its N-terminal amine or the lysine side-chain at position 6 . In the context of the localisation of the FK1TMD, it is interesting to note that, within DLPC bilayers, no higher oligomeric states are observed. Similar results were observed at a 1,000:1 ratio (data not shown), indicating that the formation of FK1TMD dimers does not appear to vary significantly with concentration within the bilayer. All experiments were repeated with $0.05 \%$ glutaraldehyde (data not shown) with results identical to those observed with DSS.

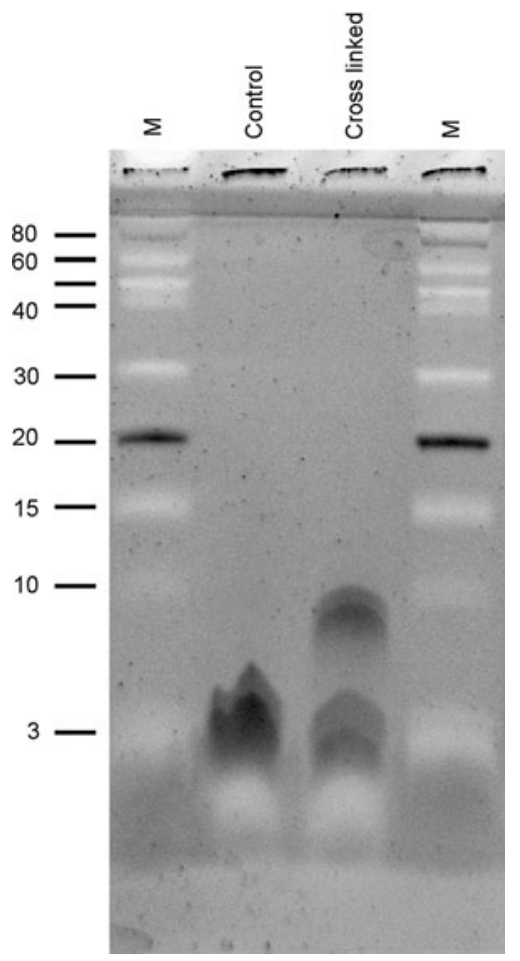

Fig. 1 Analysis of chemical cross-linking of reconstituted FK1TMD peptide using SDS-polyacrylamide gel electrophoresis (PAGE). FK1TMD was reconstituted into DLPC vesicles at lipid-to-peptide molar ratio of 100:1 and cross-linked using DSS. Cross-linked and uncrossed samples (containing $\sim 7 \mu \mathrm{g}$ FK1TMD) were run on $16 \%$ tricine SDS-PAGE gel and visualised after staining with SYPRO Orange. Cross-linked sample was visualised as two distinct bands: monomer (unlinked) and dimer. Similar results were obtained using $0.05 \%$ glutaraldehyde as cross-linker

\section{Fluorescence resonance energy transfer experiments}

To corroborate this finding, FRET measurements were made between FK1TMD labelled with FITC and RBITC as the donor and acceptor pair, respectively. FRET is a widely used technique that employs non-radiative transfer of energy between a donor and acceptor fluorophore whose emission and excitation spectra respectively overlap. Accordingly, when the two fluorophores are brought into close proximity, FRET results in a decrease in donor emission and an increase in acceptor fluorescence. The transfer efficiency function $(E)$ between the acceptor and donor fluorophore is

$E=\frac{1}{\left[1+\left(\frac{r}{R_{0}}\right)^{6}\right]}$,

where $r$ is the separation between the fluorophores and $R_{0}$ is the distance at which $50 \%$ quenching is observed and differs for individual pairs of fluorophores, which in the case of FITC and RBITC is approximately $50 \AA$ (Yegneswaran et al. 1997). Accordingly, the formation of 
oligomeric complexes will result in a theoretical quenching of up to 95-99\% should the two labelled peptides form an antiparallel to parallel dimer within the lipid bilayer, assuming a closest approach of the dimers of $\sim 10 \AA$ and a homogeneous population of donor-acceptor pairs. This property makes FRET an excellent tool for probing the oligomeric state of proteins.

To ascertain the degree of self-association and characterise the oligomeric state of FK1TMD in DLPC bilayers, the quenching of the donor, FITC-labelled FK1TMD, was monitored in the presence of increasing concentration of the acceptor RBITC-labelled FK1TMD whilst maintaining a constant lipid-to-protein ratio of 100:1 with unlabelled protein. The emission spectra are shown in Fig. 2 with the resulting fluorescence quenching plotted in Fig. 3 as a percentage of the unquenched intensity. A significant reduction in FITC-labelled FK1TMD emission is observed at $519 \mathrm{~nm}$ with increasing concentration of the acceptor RBITC-labelled FK1TMD. As expected, a corresponding increase in emission intensity is observed at $575 \mathrm{~nm}$, arising from FRET to the acceptor RBITC-labelled FK1TMD. These observations are consistent with the formation of oligomeric complexes within the bilayer containing both FITC- and RBITC-labelled FK1TMD. Control experiments exciting at $494 \mathrm{~nm}$ revealed no background emission from reconstituted unlabelled peptide or any contribution from RBITC-labelled FK1TMD at $519 \mathrm{~nm}$, although intensity was observed at longer wavelengths $(580 \mathrm{~nm})$ as shown in Fig. 2.

The number of peptides in the oligomer can be determined through analysis of the quench curve in Fig. 3. As described by Adair and Engelman (1994), the observed

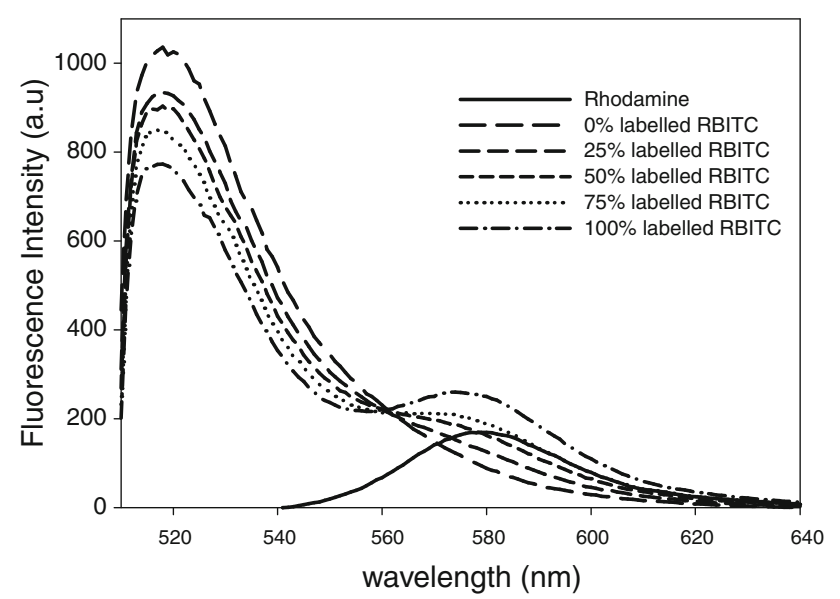

Fig. 2 Fluorescence emission spectra of FK1TMD in DLPC bilayers with constant lipid-to-peptide ratio of 100:1 (solid lines). The ratio of FITC-labelled FK1TMD to RBITC-labelled and unlabelled FK1TMD was maintained at 1:3, whilst the ratio of RBITC-labelled FK1TMD and unlabelled peptide was varied from $0 \%$ to $100 \%$ in steps of $25 \%$. Rhodamine emission spectra follow excitation at $492 \mathrm{~nm}$ showing no emission at $520 \mathrm{~nm}$ (solid line)

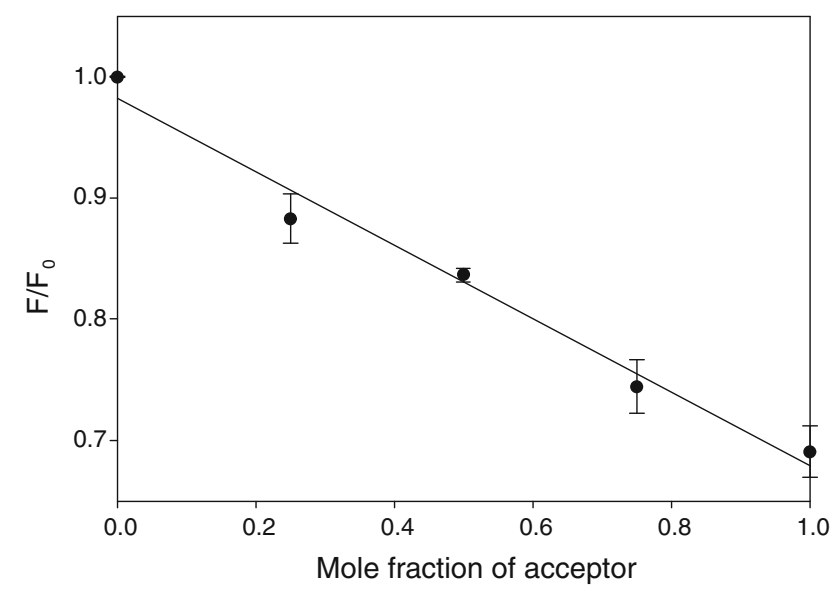

Fig. 3 Observed quenching of emission spectrum from FITClabelled FK1TMD at $520 \mathrm{~nm}$ in response to increase concentrations of the FRET acceptor RBITC-labelled FK1TMD within the DLPC bilayers (lipid-to-peptide ration, 100:1). The ratio of FITC-labelled FK1TMD to RBITC-labelled and unlabelled FK1TMD was maintained at 1:3, whilst the ratio of RBITC-labelled FK1TMD and unlabelled peptide was varied from $0 \%$ to $100 \%$. Data normalised to give $F / F_{0}$ of 1.0 in the absence of any RBITC-labelled FK1TMD acceptor. Line indicates best fit $\left(r^{2}=0.98\right)$ to the linear quenching dependency characteristic of dimer formation

quenching will be proportional to $1-\left(P_{\mathrm{FITC}}\right)^{n-1}$, where $P_{\text {FITC }}$ is the mole ratio of FITC-labelled peptide and $n$ is the number of peptides within an oligomer. Fitting the data in Fig. 3 to the linear relationship for the fluorescence intensity, $1-a P_{\mathrm{RBITC}}$, expected for a dimer $(n=2)$ gives an excellent fit with $R^{2}$ of 0.98 , significantly better than for models assuming a higher oligomeric state and in good agreement with the cross-linking data presented above.

Coarse-grained molecular dynamics simulation of FK1TMD dimerisation

Five independent simulations of FK1TMD each of duration $2 \mu$ s were performed starting from two isolated helices inserted in parallel fashion, and with similar orientations at inter-helical distance of $\sim 25 \mathrm{~nm}$ in a preformed DLPC bilayer. The minimum distance between the centres of mass of the helices was monitored as an indicator of dimerisation. In each of the five simulations a dimer was formed within $0.4 \mu \mathrm{s}$. In four cases the dimer was formed well before this, after $\sim 0.2 \mu \mathrm{s}$. In these four simulations, once formed, the dimers remained stable throughout the simulations. In one simulation we observed a dimer to dissociate into individual monomers after $\sim 0.25 \mu \mathrm{s}$; however, the dimer subsequently reformed over the next $0.1 \mu \mathrm{s}$ and thereafter remained stable throughout the remainder of the simulation (Fig. 4). In each simulation the initially parallel monomers rotated to form the dimer such that residues V8, L12, L13, T16, F20, F23, Y26 and Y27 
Fig. 4 CG simulations of dimer formation. The panel on the left shows the inter-helical distance as a function of time during the five CG simulations. In each case the dimer is stable after $0.5 \mu \mathrm{s}$. A representative snapshot of the individual monomers at the start of the simulation and a dimer are shown in backbone format (purple). The image on the right shows the dimer embedded within a DLPC lipid bilayer (lipids are green). Some residues with high propensity to interact with lipids are shown in space-filling format (R3 and K6 are cyan, $\mathrm{K} 29$ and $\mathrm{R} 35$ are orange)

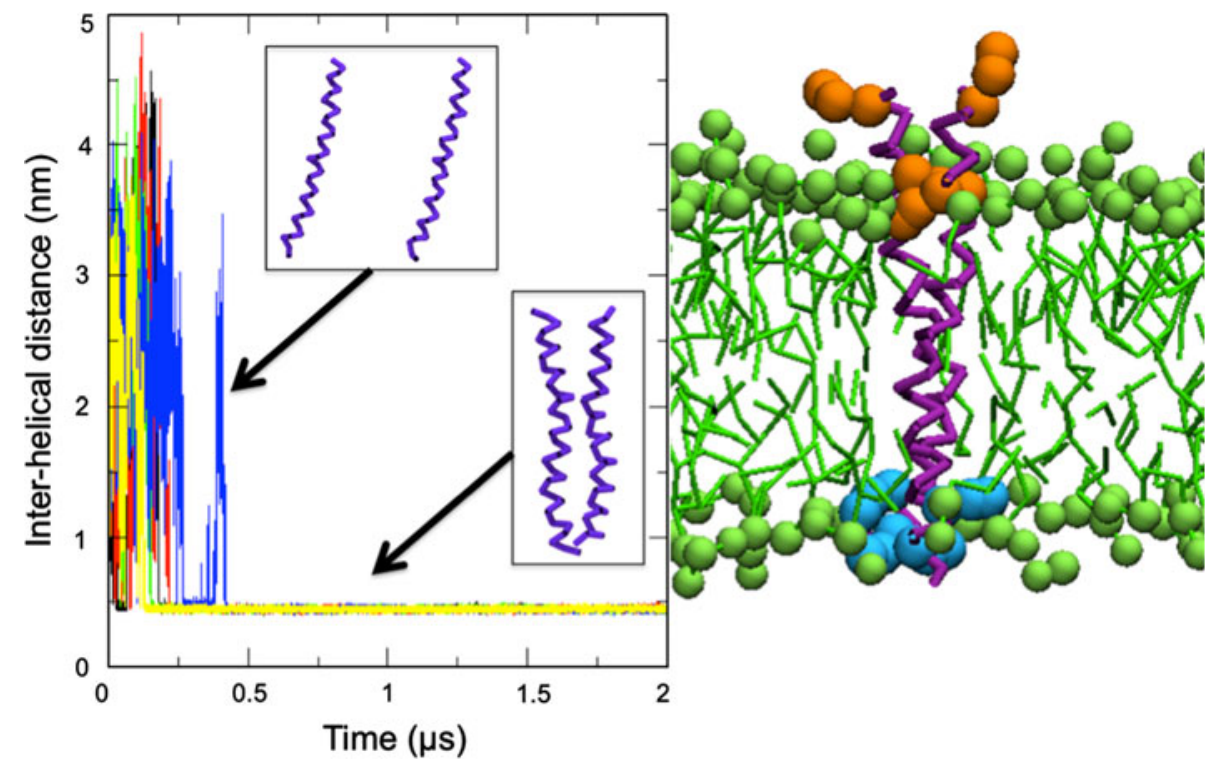

were pointing towards the membranes whereas, e.g., residues S17 and S18 were pointing 'inwards', towards the dimer interface.

Our previous simulations of isolated FK1TMD indicated that the transmembrane domain of the protein is able to avoid hydrophobic mismatch with the surrounding lipids by virtue of its ability to tilt appropriately (Holdbrook et al. 2010). It is of interest to explore whether dimerisation affects the ability of the protein to tilt within the bilayer.

The tilt angle of the individual monomers (averaged over five simulations), prior to dimerisation, with respect to the bilayer plane from the five simulations is $38.8 \pm 8.0^{\circ}$, as expected from our previous study on FK1TMD monomers in which we reported tilt angles of $39.0 \pm 11.0^{\circ}$ in DLPC (Holdbrook et al. 2010). Interestingly, after dimerisation, this angle is substantially reduced; the tilt angle for the individual monomers is $18.0 \pm 10.0^{\circ}$. These results suggest that the ability of the helices to tilt in the bilayer is affected by dimerisation, presumably due to one face of each helix being involved in protein-protein interactions and therefore not being available for protein-bilayer interactions.

To gain an understanding of the molecular interactions that play a role in anchoring the FK1TMD dimer within the lipid bilayer we analysed the specific lipid-protein contacts. Previously, our simulations of isolated FK1TMD helices in DLPC and other lipids showed that the lipids with the greatest propensity to interact with lipid headgroup particles are residues R3 and $\mathrm{K} 6$ at the N-terminal end and residues K29, R35 and N36 at the C-terminal end. In the present simulations, the dimer exhibits similar patterns of interactions with lipids. We calculated all instances of protein-lipid contact (where 'contact' is defined as $r<6 \AA$ ) in our simulations. Residues R3 and K6 at the
N-terminal accounted for $\sim 13 \%$ of all the lipid (head group)-protein contacts in all three of the simulations. At the C-terminal end, residues K29, R35 and N36 collectively make up $\sim 12 \%$ of all contacts with lipids. Thus, it is likely that these residues are responsible for anchoring the dimer in the membrane. In all cases the contacts between lipid and proteins are predominantly between the phosphate moiety of the lipid head group and the amino acid side-chains. Thus, it is likely that residues R3, K6, K29, R35 and N26 are responsible for anchoring the dimer in the membrane.

\section{Atomistic refinement of the FK1TMD dimer structure}

To further explore the conformational stability and dynamics of the FK1TMD dimer, we converted the CG dimer to atomistic detail using the protocol described in Stansfeld and Sansom (2011). The atomistic resolution enables identification of specific protein-protein interactions that play a role in stabilising the dimer. The dimer was manually embedded within a DLPC bilayer (the bilayer lipids were also converted to atomistic resolution from our own CG simulations) using the CG simulations as a guide in terms of its location relative to the lipid head groups. Three independent simulations, each of duration $100 \mathrm{~ns}$, were performed to refine the protein-protein interface of the FK1TMD dimer.

In agreement with the CG simulations, the helices remained in parallel orientation with respect to each other. The secondary structure of the dimer helices was evaluated initially by visual inspection. The helices were generally stable throughout the simulations with some unfolding $(\sim 3$ residues) observed at the termini. The secondary structure was quantitatively evaluated with the DSSP code, which 
Fig. 5 Inter-helix interactions of residues $\mathrm{S} 17$ and $\mathrm{S} 18$. The plot on the left shows the distance between these residues as a function of time in the three atomistic simulations. A representative snapshot of the dimer backbone (purple) is shown on the right. Residues S17 and S18 are shown in space-filling format (carbon atoms are cyan, nitrogen atoms are blue, oxygen atoms are red and polar hydrogen atoms are white). The same residues are shown close-up in stick representation in the bottom right panel, with hydrogen bonds shown in green

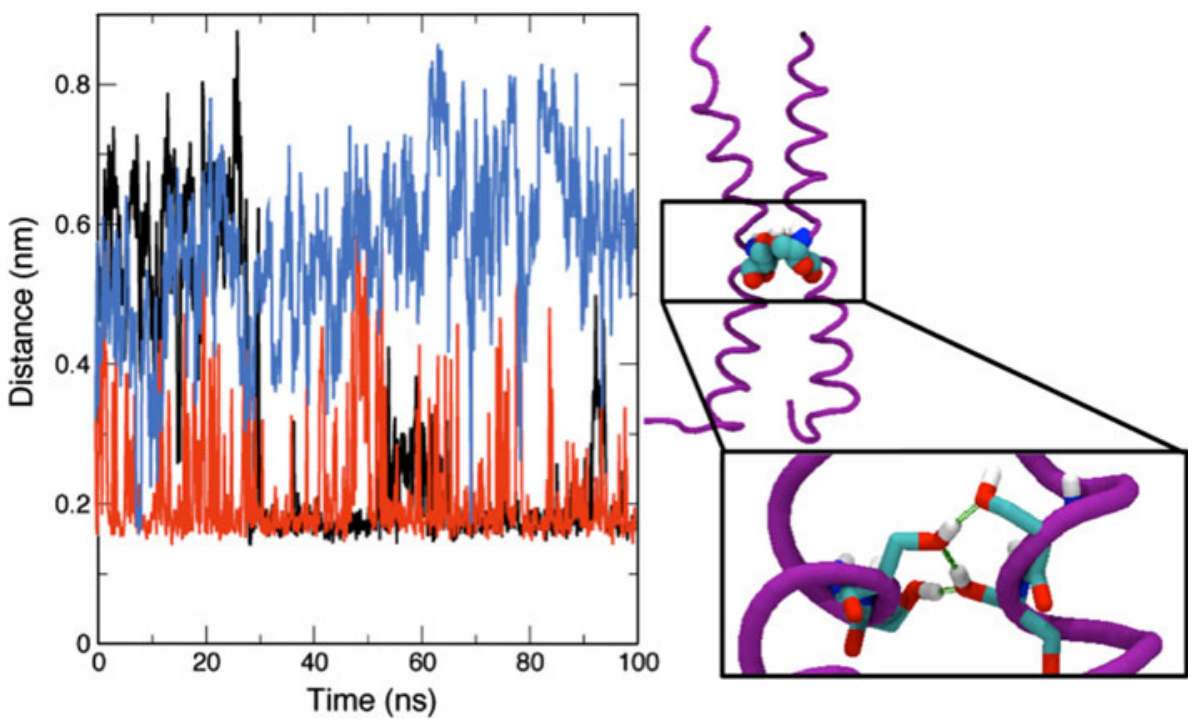

confirmed the presence of stable $\alpha$-helices throughout the simulations (see Supplementary Information).

We evaluated the dimer interface by calculating the number of interactions between residues of the two FK1TMD helices. Here we define interaction as inter-residue distance of $\leq 0.35 \mathrm{~nm}$. The most persistent interactions arose from the TXXSS motif, with interactions between these residues present in all three simulations (Fig. 5). In particular, hydrogen bonding was observed between residues S17-S18 and S18-S18, with some hydrogen bonds formed for up to $\sim 79 \%$ of the simulations (full details in Supplementary Table 2). The hydrogen bonds were formed between the hydroxyl groups of the residue side-chains. Whilst there are interactions between the residues of this motif in all three simulations, not all the interactions are present all of the time, indicating some flexibility in this region. Furthermore, the close proximity of residues S17 and $\mathrm{S} 18$, in comparison with the other helix residues, caused the dimer to kink slightly in this region, near the centre of the helices (Fig. 5). The side-chain of T16 was orientated towards the lipid bilayer, thus this residue did not provide a major contribution to inter-helix interactions. We did not observe any persistent hydrogen-bonding interactions or salt bridges at either dimer terminus.

The atomistic-level detail also enabled more detailed evaluation of the protein-lipid contacts that we had observed in the CG simulations. In agreement with the CG simulations, the majority of interactions with the lipid head groups were with residues R3, K6, K29, R35 and N26. Both hydrogen-bonding and charge-charge interactions were observed. The guanidinium and amino moieties of the arginine and lysine residues, respectively, are observed to orientate themselves such that they are pointing towards the phosphate groups of the DLPC lipids (Fig. 6). As a consequence of the combined electrostatic and hydrogenbonding interactions, individual lipids are observed to remain in contact with the protein (where contact is defined as protein distance $<3.5 \AA$ ) on timescales of up to $40 \mathrm{~ns}$

Fig. 6 Examples of lipidprotein interactions. The panel on the left shows an electrostatic interaction between the guanidinium group R3 of a Fukutin monomer and a phosphate group of a DLPC lipid molecule. The panel on the right shows a hydrogen-bond interaction between $\mathrm{N} 5$ of a Fukutin monomer and a phosphate group of a DLPC lipid

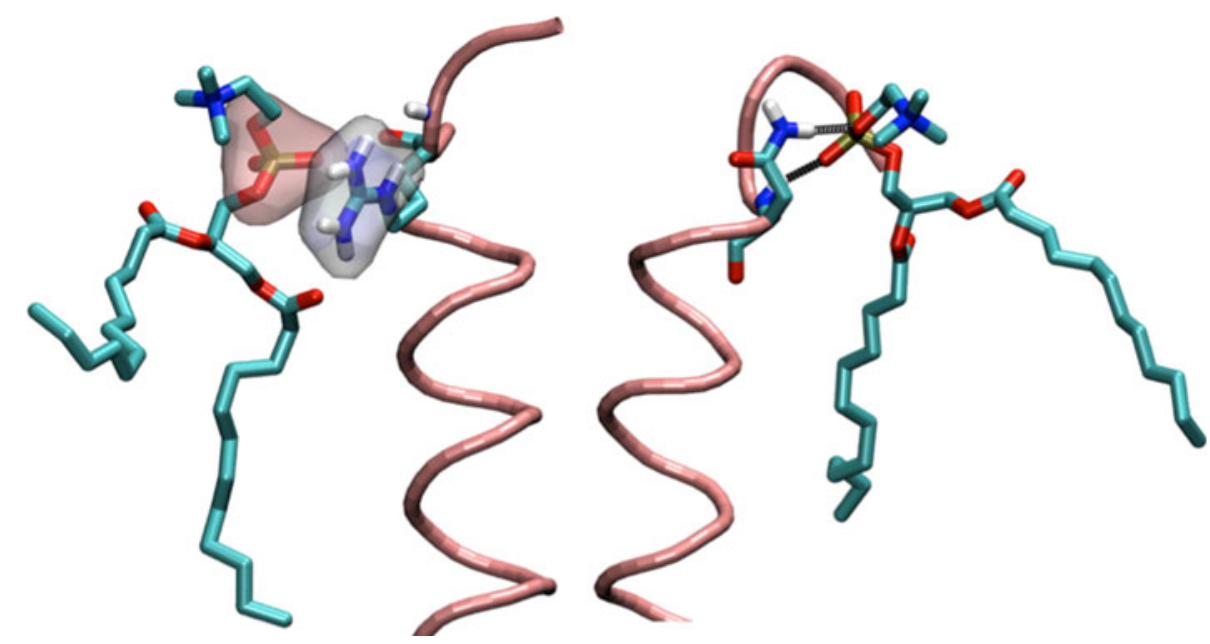


(see Supplementary Information). All of the residues R3, K6, K29, R35 and N26 were observed to participate in hydrogen bonding with lipid head groups.

\section{Discussion}

We employed chemical cross-linking and fluorescence energy transfer measurements to determine the oligomeric state of FK1TMD within DLPC bilayers to be dimeric with no evidence of higher oligomeric species. This finding was corroborated in silico using coarse-grained molecular dynamics (CGMD) simulations that demonstrated the propensity of FK1TMD to form stable dimeric complexes in DLPC lipid bilayers. CG and atomistic simulations identified residues at both termini of the Fukutin-I helix that engage in hydrogen bonding and charge-charge interactions with lipid head groups. While N26 interacts with lipids via hydrogen bonds alone, the charged residues R3, K6, K29 and R35 are observed to form hydrogen bonds and charge-charge interactions with lipid head groups, these interactions essentially serving to anchor the helix within the lipid bilayer. The formation of the dimeric species resulted in a significant reduction in the tilt of the helix with respect to the bilayer normal. With little evidence of bilayer deformation surrounding the dimer to accommodate the reduction in tilt angle, it appears that the loss of electrostatic and hydrogen-bonding interactions between lipid and protein is compensated by inter-helix hydrogen bonding and the slight reduction in the width of the hydrophobic membrane spanning domain of the peptide arising from the formation of a kink at the TXXSS motif. This reduction in the hydrophobic width of the helices was observed to arise from the formation of a kink at the TXXSS motif. Atomistic simulations revealed the TXXSS motif to play a major role in the stability of the dimer, being the major contributor of inter-helical hydrogen bonds. The TXXSS-type motif identified here has previously been identified as playing an important role promoting interactions between transmembrane helices (Dawson et al. 2002). Interestingly, sequence analysis revealed that other members of this family of glycosyltransferases are devoid of this motif, which may have implications for the hetero-oligomerisation of Fukutin-I with other members of this family of glycosyltransferases, impacting on its potential role in intra-cellular localisation and the resulting colocalisation of enzymatic activity.

\section{Conclusions}

A combination of experimental and molecular simulation techniques was employed to study the oligomerisation of the N-terminal transmembrane domain of Fukutin-I. We determined that the peptide exists as dimer in DLPC lipid bilayers, with the TXXSS motif playing a key role in maintaining the dimeric state. In DLPC lipids, the protein is observed to tilt rather than deform the bilayer to avoid hydrophobic mismatch. The extent of tilting is reduced after dimerisation, presumably due to fewer residues being exposed to the hydrophobic core of the bilayer. As the formation of higher oligomeric species has been implicated in the retention of Fukutin-I and other proteins within the ER/GA, a natural extension of the current studies is to explore the oligomeric state of Fukutin-I in the more complex lipid mixtures found in the ER/GA. It will be particularly illuminating to ascertain how its oligomeric state changes in response to the changing lipid composition of different intracellular compartments.

Open Access This article is distributed under the terms of the Creative Commons Attribution Noncommercial License which permits any noncommercial use, distribution, and reproduction in any medium, provided the original author(s) and source are credited.

\section{References}

Adair BD, Engelman DM (1994) Glycophorin A helical transmembrane domains dimerize in phospholipid bilayers: a resonance energy transfer study. Biochemistry 33(18):5539-5544

Berendsen HJC, Postma JPM, van Gunsteren WF, Hermans J (1981) In intermolecular forces. Reidel, Dordrecht

Berendsen H, van der Spoel D, van Drunen R (1995) GROMACS-a message passing parallel molecular-dynamics implementation. Comput Phys Comm 91:43-56

Bond PJ, Sansom MS (2006) Insertion and assembly of membrane proteins via simulation. J Am Chem Soc 128(8):2697-2704

Bond PJ, Holyoake J, Ivetac A, Khalid S, Sansom MS (2007) Coarsegrained molecular dynamics simulations of membrane proteins and peptides. J Struct Biol 157(3):593-605

Bond PJ, Wee CL, Sansom MSP (2008) Coarse-Grained Molecular Dynamics Simulations of the Energetics of Helix Insertion into a Lipid Bilayer. Biochemistry 47(43):11321-11331

Carpenter T, Bond PJ, Khalid S, Sansom MS (2008) Self-assembly of a simple membrane protein: coarse-grained molecular dynamics simulations of the influenza M2 channel. Biophys J 95(8): 3790-3801

Dawson JP, Weinger JS, Engelman DM (2002) Motifs of serine and threonine can drive association of transmembrane helices. J Mol Biol 316(3):799-805

Esapa CT, McIlhinney RA, Blake DJ (2005) Fukutin-related protein mutations that cause congenital muscular dystrophy result in ERretention of the mutant protein in cultured cells. Hum Mol Genet 14(2):295-305

Essmann U, Perera L, Berkowitz ML, Darden T, Lee H, Pedersen LG (1995) A smooth particle mesh Ewald method. J Chem Phys 103(19):8577-8593

Gallova J, Uhrikova D, Islamov A, Kuklin A, Balgavy P (2004) Effect of cholesterol on the bilayer thickness in unilamellar extruded DLPC and DOPC liposomes: SANS contrast variation study. Gen Physiol Biophys 23(1):113-128

Gleeson PA (1998) Targeting of proteins to the Golgi apparatus. Histochem Cell Biol 109(5-6):517-532 
Hess B (2008) P-LINCS: a parallel linear constraint solver for molecular simulation. J Chem Theory Comput 4(1):116-122

Hess B, Kutzner C, van der Spoel D, Lindahl E (2008) GROMACS 4: algorithms for highly efficient, load-balanced, and scalable molecular simulation. J Chem Theory Comput 4:435-447

Holdbrook DA, Leung YM, Piggot TJ, Marius P, Williamson PT, Khalid S (2010) Stability and membrane orientation of the fukutin transmembrane domain: a combined multiscale molecular dynamics and circular dichroism study. Biochemistry 49(51):10796-10802

Hoover WG (1985) Canonical dynamics: equilibrium phase-space distributions. Phys Rev A 31(3):1695-1697

Humphrey W, Dalke A, Schulten K (1996) VMD: visual molecular dynamics. J Mol Graphics 14(1):33-38

Kabsch W, Sander C (1983) Dictionary of protein secondary structure-pattern-recognition of hydrogen-bonded and geometrical features. Biopolymers 22(12):2577-2637

Keramaris-Vrantsis E, Lu PJ, Doran T, Zillmer A, Ashar J, Esapa CT, Benson MA, Blake DJ, Rosenfeld J, Lu QL (2007) Fukutinrelated protein localizes to the Golgi apparatus and mutations lead to mislocalization in muscle in vivo. Muscle Nerve 36(4):455-465

Kukol A (2009) Lipid models for united-atom molecular dynamics simulations of proteins. J Chem Theory Comput 5(3):615-626

Laskowski RA, Moss DS, Thornton JM (1993) Main-chain bond lengths and bond angles in protein structures. $\mathrm{J}$ Mol Biol 231(4):1049-1067

Lommel M, Willer T, Strahl S (2008) POMT2, a key enzyme in Walker-Warburg syndrome: somatic sPOMT2, but not testis specific POMT2, is crucial for mannosyltransferase activity in vivo. Glycobiology 18:615-625

Marius P, Wright JN, Findlow IS, Williamson PT (2010) Expression and purification of the transmembrane domain of Fukutin-I for biophysical studies. Protein Expr Purif 72:107-112

Marrink SJ, de Vries AH, Mark AE (2004) Coarse grained model for semiquantitative lipid simulations. J Phys Chem B 108(2): $750-760$

Martin-Rendon E, Blake DJ (2003) Protein glycosylation in disease: new insights into the congenital muscular dystrophies. Trends Pharmacol Sci 24(4):178-183

Marti-Renom MA, Stuart AC, Fiser A, Sanchez R, Melo F, Sali A (2000) Comparative protein structure modeling of genes and genomes. Annu Rev Biophys Biomol Struct 29:291-325
Matsumoto H, Noguchi S, Sugie K, Ogawa M, Murayama K, Hayashi YK, Nishino I (2004) Subcellular localization of fukutin and fukutin-related protein in muscle cells. J Biochem 135(6):709-712

Mitra K, Ubarretxena-Belandia I, Taguchi T, Warren G, Engelman DM (2004) Modulation of the bilayer thickness of exocytic pathway membranes by membrane proteins rather than cholesterol. Proc Natl Acad Sci USA 101(12):4083-4088

Miyamoto S, Kollman PA (1992) Settle-an analytical version of the shake and rattle algorithm for rigid water models. J Comput Chem 13(8):952-962

Munro S (1998) Localization of proteins to the Golgi apparatus. Trends Cell Biol 8:11-15

Oostenbrink C, Villa A, Mark AE, van Gunsteren WF (2004) A biomolecular force field based on the free enthalpy of hydration and solvation: the GROMOS force-field parameter sets 53A5 and 53A6. J Comput Chem 25(13):1656-1676

Opat AS, van Vliet C, Gleeson PA (2001) Trafficking and localisation of resident Golgi glycosylation enzymes. Biochimie 83(8):763773

Parrinello M, Rahman A (1981) Polymorphic transitions in single crystals: a new molecular dynamics method. J Appl Phys 52(12):7182-7190

Pelham HRB, Munro S (1993) Sorting of membrane proteins in the secretory pathway. Cell 75:603-605

Rayner JC, Pelham HRB (1997) Transmembrane domain-dependent sorting of proteins to the ER and plasma membrane in yeast. EMBO J 16(8):1832-1841

Stansfeld PJ, Sansom MSP (2011) From coarse grained to atomistic: a serial multiscale approach to membrane protein simulations. J Chem Theory Comput 7(4):1157-1166

Torelli S, Brown SC, Brockington M, Dolatshad NF, Jimenez C, Skordis L, Feng LH, Merlini L, Jones DH, Romero N, Wewer U, Voit T, Sewry CA, Noguchi S, Nishino I, Muntoni F (2005) Subcellular localisation of fukutin related protein in different cell lines and in the muscle patients with MDC1C and LGMD2I. Neuromuscul Disord 15:836-843

van der Spoel D, Lindahl E, Hess B, Groenhof G, Mark AE, Berendsen HJC (2005) GROMACS: fast, flexible, and free. J Comput Chem 26:1701-1718

Yegneswaran S, Wood GM, Esmon CT, Johnson AE (1997) Protein S alters the active site location of activated protein $\mathrm{C}$ above the membrane surface. A fluorescence resonance energy transfer study of topography. J Biol Chem 272(40):25013-25021 